\title{
Boundedness of a Class of Sublinear Operators and Their Commutators on Generalized Morrey Spaces
}

\author{
Vagif S. Guliyev, ${ }^{1,2}$ Seymur S. Aliyev, ${ }^{2}$ and Turhan Karaman ${ }^{1}$ \\ ${ }^{1}$ Department of Mathematics, Ahi Evran University, 40100 Kirsehir, Turkey \\ ${ }^{2}$ Institute of Mathematics and Mechanics of NAS of Azerbaijan, Baku 1141, Azerbaijan \\ Correspondence should be addressed to Vagif S. Guliyev, vagif@guliyev.com
}

Received 10 April 2011; Revised 23 April 2011; Accepted 18 May 2011

Academic Editor: Irena Lasiecka

Copyright (C) 2011 Vagif S. Guliyev et al. This is an open access article distributed under the Creative Commons Attribution License, which permits unrestricted use, distribution, and reproduction in any medium, provided the original work is properly cited.

The authors study the boundedness for a large class of sublinear operator $T$ generated by Calderon-Zygmund operator on generalized Morrey spaces $M_{p, \varphi}$. As an application of this result, the boundedness of the commutator of sublinear operators $T_{a}$ on generalized Morrey spaces is obtained. In the case $a \in \mathrm{BMO}\left(\mathbb{R}^{n}\right), 1<p<\infty$ and $T_{a}$ is a sublinear operator, we find the sufficient conditions on the pair $\left(\varphi_{1}, \varphi_{2}\right)$ which ensures the boundedness of the operator $T_{a}$ from one generalized Morrey space $M_{p, \varphi_{1}}$ to another $M_{p, \varphi_{2}}$. In all cases, the conditions for the boundedness of $T_{a}$ are given in terms of Zygmund-type integral inequalities on $\left(\varphi_{1}, \varphi_{2}\right)$, which do not assume any assumption on monotonicity of $\varphi_{1}, \varphi_{2}$ in $r$. Conditions of these theorems are satisfied by many important operators in analysis, in particular pseudodifferential operators, Littlewood-Paley operator, Marcinkiewicz operator, and Bochner-Riesz operator.

\section{Introduction}

For $x \in \mathbb{R}^{n}$ and $r>0$, we denote by $B(x, r)$ the open ball centered at $x$ of radius $r$, and by ${ }^{\complement} B(x, r)$ denote its complement. Let $|B(x, r)|$ be the Lebesgue measure of the ball $B(x, r)$.

Let $f \in L_{1}^{\text {loc }}\left(\mathbb{R}^{n}\right)$. The Hardy-Littlewood maximal operator $M$ is defined by

$$
M f(x)=\sup _{t>0}|B(x, t)|^{-1} \int_{B(x, t)}|f(y)| d y
$$

Let $K$ be a Calderón-Zygmund singular integral operator, briefly a Calderón-Zygmund operator, that is, a linear operator bounded from $L_{2}\left(\mathbb{R}^{n}\right)$ to $L_{2}\left(\mathbb{R}^{n}\right)$ taking all infinitely 
continuously differentiable functions $f$ with compact support to the functions $f \in L_{1}^{\text {loc }}\left(\mathbb{R}^{n}\right)$ represented by

$$
K f(x)=\int_{\mathbb{R}^{n}} k(x, y) f(y) d y \quad x \notin \operatorname{supp} f .
$$

Such operators were introduced in [1]. Here, $k(x, y)$ is a continuous function away from the diagonal which satisfies the standard estimates: there exist $c_{1}>0$ and $0<\varepsilon \leq 1$ such that

$$
|k(x, y)| \leq c_{1}|x-y|^{-n}
$$

for all $x, y \in \mathbb{R}^{n}, x \neq y$, and

$$
\left|k(x, y)-k\left(x^{\prime}, y\right)\right|+\left|k(y, x)-k\left(y, x^{\prime}\right)\right| \leq c_{1}\left(\frac{\left|x-x^{\prime}\right|}{|x-y|}\right)^{\varepsilon}|x-y|^{-n}
$$

whenever $2\left|x-x^{\prime}\right| \leq|x-y|$.

It is well known that maximal operator and Calderón-Zygmund operators play an important role in harmonic analysis (see [2-6]).

Suppose that $T$ represents a linear or a sublinear operator, which satisfies that for any $f \in L_{1}\left(\mathbb{R}^{n}\right)$ with compact support and $x \notin \operatorname{supp} f$

$$
|T f(x)| \leq c_{0} \int_{\mathbb{R}^{n}} \frac{|f(y)|}{|x-y|^{n}} d y
$$

where $c_{0}$ is independent of $f$ and $x$.

For a function $a$, suppose that the commutator operator $T_{a}$ represents a linear or a sublinear operator, which satisfies that for any $f \in L_{1}\left(\mathbb{R}^{n}\right)$ with compact support and $x \notin \operatorname{supp} f$

$$
\left|T_{a} f(x)\right| \leq c_{0} \int_{\mathbb{R}^{n}}|a(x)-a(y)||x-y|^{-n}|f(y)| d y,
$$

where $c_{0}$ is independent of $f$ and $x$.

We point out that the condition (1.5) was first introduced by Soria and Weiss in [7]. The condition (1.5) are satisfied by many interesting operators in harmonic analysis, such as the Calderón-Zygmund operators, Carleson's maximal operator, Hardy-Littlewood maximal operator, C. Fefferman's singular multipliers, R. Fefferman's singular integrals, Ricci-Stein's oscillatory singular integrals, and the Bochner-Riesz means (see $[7,8]$ for details).

In this work, we prove the boundedness of the sublinear operator $T$ satisfies the condition (1.5) generated by Calderon-Zygmund operator from one generalized Morrey space $M_{p, \varphi_{1}}$ to another $M_{p, \varphi_{2}}, 1<p<\infty$, and from $M_{1, \varphi_{1}}$ to the weak space $W M_{1, \varphi_{2}}$. In the case $a \in \mathrm{BMO}\left(\mathbb{R}^{n}\right), 1<p<\infty$ and the commutator operator $T_{a}$ is a sublinear operator, we find the sufficient conditions on the pair $\left(\varphi_{1}, \varphi_{2}\right)$ which ensures the boundedness of the operators $T_{a}$ from $M_{p, \varphi_{1}}$ to $M_{p, \varphi_{2}}$. Finally, as applications, we apply this result to several 
particular operators such as the pseudodifferential operators, Littlewood-Paley operator, Marcinkiewicz operator, and Bochner-Riesz operator.

By $A \lesssim B$, we mean that $A \leq C B$ with some positive constant $C$ independent of appropriate quantities. If $A \lesssim B$ and $B \lesssim A$, we write $A \approx B$ and say that $A$ and $B$ are equivalent.

\section{Morrey Spaces}

The classical Morrey spaces $M_{p, \lambda}$ were originally introduced by Morrey Jr. in [9] to study the local behavior of solutions to second-order elliptic partial differential equations. For the properties and applications of classical Morrey spaces, we refer the readers to $[9,10]$.

We denote by $M_{p, \lambda} \equiv M_{p, \lambda}\left(\mathbb{R}^{n}\right)$ the Morrey space, the space of all functions $f \in$ $L_{p}^{\text {loc }}\left(\mathbb{R}^{n}\right)$ with finite quasinorm

$$
\|f\|_{M_{p, \lambda}} \equiv\|f\|_{M_{p, \lambda}\left(\mathbb{R}^{n}\right)}=\sup _{x \in \mathbb{R}^{n}, r>0} r^{-\lambda / p}\|f\|_{L_{p}(B(x, r))^{\prime}}
$$

where $1 \leq p<\infty$ and $0 \leq \lambda \leq n$.

Note that $M_{p, 0}=L_{p}\left(\mathbb{R}^{n}\right)$ and $M_{p, n}=L_{\infty}\left(\mathbb{R}^{n}\right)$. If $\lambda<0$ or $\lambda>n$, then $M_{p, \lambda}=\Theta$, where $\Theta$ is the set of all functions equivalent to 0 on $\mathbb{R}^{n}$.

We also denote by $W M_{p, \lambda} \equiv W M_{p, \lambda}\left(\mathbb{R}^{n}\right)$ the weak Morrey space of all functions $f \in$ $W L_{p}^{\text {loc }}\left(\mathbb{R}^{n}\right)$ for which

$$
\|f\|_{W M_{p, \lambda}} \equiv\|f\|_{W M_{p, \lambda}\left(\mathbb{R}^{n}\right)}=\sup _{x \in \mathbb{R}^{n}, r>0} r^{-\lambda / p}\|f\|_{W L_{p}(B(x, r))}<\infty
$$

where $W L_{p}(B(x, r))$ denotes the weak $L_{p}$-space of measurable functions $f$ for which

$$
\begin{aligned}
\|f\|_{W L_{p}(B(x, r))} & \equiv\left\|f X_{B(x, r)}\right\|_{W L_{p}\left(\mathbb{R}^{n}\right)} \\
& =\sup _{t>0} t|\{y \in B(x, r):|f(y)|>t\}|^{1 / p} \\
& =\sup _{0<t \leq|B(x, r)|} t^{1 / p}\left(f_{X_{B}(x, r)}\right)^{*}(t)<\infty .
\end{aligned}
$$

Here, $g^{*}$ denotes the nonincreasing rearrangement of a function $g$.

Chiarenza and Frasca [11] studied the boundedness of the maximal operator $M$ in these spaces. Their result can be summarized as follows.

Theorem 2.1. Let $1 \leq p<\infty$ and $0 \leq \lambda<n$. Then, for $p>1$ the operator $M$ is bounded on $M_{p, \lambda}$ and for $p=1 M$ is bounded from $M_{1, \lambda}$ to $W M_{1, \lambda}$.

Di Fazio and Ragusa [12] studied the boundedness of the Calderon-Zygmund operators in Morrey spaces, and their results imply the following statement for CalderonZygmund operators $K$.

Theorem 2.2. Let $1 \leq p<\infty, 0<\lambda<n$. Then, for $1<p<\infty$ Calderon-Zygmund operator $K$ is bounded on $M_{p, \lambda}$ and for $p=1 \mathrm{~K}$ is bounded from $M_{1, \lambda}$ to $W M_{1, \lambda}$. 
Note that Theorem 2.2 was proved by Peetre [10] in the case of the classical CalderonZygmund singular integral operators.

\section{Generalized Morrey Spaces}

We find it convenient to define the generalized Morrey spaces in the form as follows.

Definition 3.1. Let $\varphi(x, r)$ be a positive measurable function on $\mathbb{R}^{n} \times(0, \infty)$ and $1 \leq p<\infty$. We denote by $M_{p, \varphi} \equiv M_{p, \varphi}\left(\mathbb{R}^{n}\right)$ the generalized Morrey space, the space of all functions $f \in$ $L_{p}^{\text {loc }}\left(\mathbb{R}^{n}\right)$ with finite quasinorm

$$
\|f\|_{M_{p, \varphi}} \equiv\|f\|_{M_{p, \varphi}\left(\mathbb{R}^{n}\right)}=\sup _{x \in \mathbb{R}^{n}, r>0} \varphi(x, r)^{-1}|B(x, r)|^{-1 / p}\|f\|_{L_{p}(B(x, r))} .
$$

Also, by $W M_{p, \varphi} \equiv W M_{p, \varphi}\left(\mathbb{R}^{n}\right)$ we denote the weak generalized Morrey space of all functions $f \in W L_{p}^{\text {loc }}\left(\mathbb{R}^{n}\right)$ for which

$$
\|f\|_{W M_{p, \varphi}} \equiv\|f\|_{W M_{p, \varphi}\left(\mathbb{R}^{n}\right)}=\sup _{x \in \mathbb{R}^{n}, r>0} \varphi(x, r)^{-1}|B(x, r)|^{-1 / p}\|f\|_{W L_{p}(B(x, r))}<\infty .
$$

According to this definition, we recover the spaces $M_{p, \lambda}$ and $W M_{p, \lambda}$ under the choice $\varphi(x, r)=r^{(\lambda-n) / p}:$

$$
\begin{aligned}
M_{p, \lambda} & =\left.M_{p, \varphi}\right|_{\varphi(x, r)=r^{(\lambda-n) / p},} \\
W M_{p, \lambda} & =\left.W M_{p, \varphi}\right|_{\varphi(x, r)=r^{(\lambda-n) / p}} .
\end{aligned}
$$

In [13-19], there were obtained sufficient conditions on $\varphi_{1}$ and $\varphi_{2}$ for the boundedness of the maximal operator $M$ and Calderon-Zygmund operator $K$ from $M_{p, \varphi_{1}}$ to $M_{p, \varphi_{2}}$, $1<p<\infty$ (see also [20-23]). In [19], the following condition was imposed on $\varphi(x, r)$ :

$$
c^{-1} \varphi(x, r) \leq \varphi(x, t) \leq c \varphi(x, r),
$$

whenever $r \leq t \leq 2 r$, where $c(\geq 1)$ does not depend on $t, r$ and $x \in \mathbb{R}^{n}$, jointly with the condition

$$
\int_{r}^{\infty} \varphi(x, t)^{p} \frac{d t}{t} \leq C \varphi(x, r)^{p}
$$

for the sublinear operator $T$ satisfies the condition (1.5), where $C(>0)$ does not depend on $r$ and $x \in \mathbb{R}^{n}$. 


\section{Sublinear Operators Generated by Calderón-Zygmund Operators in the Spaces $M_{p, \varphi}$}

In [24] (see, also $[25,26]$ ), the following statements was proved by sublinear operator $T$ satisfies the condition (1.5), containing the result in $[18,19]$.

Theorem 4.1. Let $1<p<\infty$ and $\varphi(x, r)$ satisfy conditions (3.4) and (3.5). Let $T$ be a sublinear operator satisfies the condition (1.5) and bounded on $L_{p}\left(\mathbb{R}^{n}\right)$. Then, the operator T is bounded on $M_{p, \varphi}$.

The following statements, containing results obtained in $[18,19]$ was proved in [13] (see also $[14,15])$.

Theorem 4.2. Let $1 \leq p<\infty$, and let $\left(\varphi_{1}, \varphi_{2}\right)$ satisfy the condition

$$
\int_{t}^{\infty} \varphi_{1}(x, r) \frac{d r}{r} \leq C \varphi_{2}(x, t)
$$

where $C$ does not depend on $x$ and $t$. Then, the operators $M$ and $K$ are bounded from $M_{p, \varphi_{1}}$ to $M_{p, \varphi_{2}}$ for $p>1$ and from $M_{1, \varphi_{1}}$ to $W M_{1, \varphi_{2}}$.

In this section, we are going to use the following statement on the boundedness of the Hardy operator:

$$
(H g)(t):=\frac{1}{t} \int_{0}^{t} g(r) d r, \quad 0<t<\infty
$$

Theorem 4.3 (see [27]). The inequality

$$
\underset{t>0}{\operatorname{ess} \sup } w(t) H g(t) \leq c \text { ess } \sup v(t) g(t)
$$

holds for all nonnegative and nonincreasing $g$ on $(0, \infty)$ if and only if

$$
A:=\sup _{t>0} \frac{w(t)}{t} \int_{0}^{t} \frac{d r}{\operatorname{ess} \sup _{0<s<r} v(s)}<\infty
$$

and $c \approx A$.

Lemma 4.4. Let $1 \leq p<\infty, T$ be a sublinear operator which satisfies the condition (1.5) bounded on $L_{p}\left(\mathbb{R}^{n}\right)$ for $p>1$ and bounded from $L_{1}\left(\mathbb{R}^{n}\right)$ to $W L_{1}\left(\mathbb{R}^{n}\right)$.

Then, for $1<p<\infty$,

$$
\|T f\|_{L_{p}(B)} \lesssim r^{n / p} \int_{2 r}^{\infty} t^{-n / p-1}\|f\|_{L_{p}\left(B\left(x_{0}, t\right)\right)} d t
$$

holds for any ball $B=B\left(x_{0}, r\right)$ and for all $f \in L_{p}^{\text {loc }}\left(\mathbb{R}^{n}\right)$. 
Moreover, for $p=1$,

$$
\|T f\|_{W L_{1}(B)} \lesssim r^{n} \int_{2 r}^{\infty} t^{-n-1}\|f\|_{L_{1}\left(B\left(x_{0}, t\right)\right)} d t
$$

holds for any ball $B=B\left(x_{0}, r\right)$ and for all $f \in L_{1}^{\text {loc }}\left(\mathbb{R}^{n}\right)$.

Proof. Let $p \in(1, \infty)$. For arbitrary $x_{0} \in \mathbb{R}^{n}$, set $B=B\left(x_{0}, r\right)$ for the ball centered at $x_{0}$ and of radius $r, 2 B=B\left(x_{0}, 2 r\right)$. We represent $f$ as

$$
f=f_{1}+f_{2}, \quad f_{1}(y)=f(y) \chi_{2 B}(y), \quad f_{2}(y)=f(y) \chi^{c}(2 B)(y), \quad r>0
$$

and have

$$
\|T f\|_{L_{p}(B)} \leq\left\|T f_{1}\right\|_{L_{p}(B)}+\left\|T f_{2}\right\|_{L_{p}(B)}
$$

Since $f_{1} \in L_{p}\left(\mathbb{R}^{n}\right), T f_{1} \in L_{p}\left(\mathbb{R}^{n}\right)$ and from the boundedness of $T$ in $L_{p}\left(\mathbb{R}^{n}\right)$, it follows that

$$
\left\|T f_{1}\right\|_{L_{p}(B)} \leq\left\|T f_{1}\right\|_{L_{p}\left(\mathbb{R}^{n}\right)} \leq C\left\|f_{1}\right\|_{L_{p}\left(\mathbb{R}^{n}\right)}=C\|f\|_{L_{p}(2 B)^{\prime}}
$$

where constant $C>0$ is independent of $f$.

It is clear that $x \in B, y \in{ }^{\mathrm{c}}(2 \mathrm{~B})$ implies $(1 / 2)\left|x_{0}-y\right| \leq|x-y| \leq(3 / 2)\left|x_{0}-y\right|$. We get

$$
\left|T f_{2}(x)\right| \leq 2^{n} c_{0} \int_{{ }^{c}(2 B)} \frac{|f(y)|}{\left|x_{0}-y\right|^{n}} d y .
$$

By Fubini's theorem, we have

$$
\begin{aligned}
\int_{{ }(2 B)} \frac{|f(y)|}{\left|x_{0}-y\right|^{n}} d y & \approx \int_{{ }(2 B)}|f(y)| \int_{\left|x_{0}-y\right|}^{\infty} \frac{d t}{t^{n+1}} d y \\
& \approx \int_{2 r}^{\infty} \int_{2 r \leq\left|x_{0}-y\right|<t}|f(y)| d y \frac{d t}{t^{n+1}} \\
& \lesssim \int_{2 r}^{\infty} \int_{B\left(x_{0}, t\right)}|f(y)| d y \frac{d t}{t^{n+1}}
\end{aligned}
$$

Applying Hölder's inequality, we get

$$
\int_{{ }(2 B)} \frac{|f(y)|}{\left|x_{0}-y\right|^{n}} d y \lesssim \int_{2 r}^{\infty}\|f\|_{L_{p}\left(B\left(x_{0}, t\right)\right)} \frac{d t}{t^{n / p+1}} .
$$


Abstract and Applied Analysis

Moreover, for all $p \in[1, \infty)$,

$$
\left\|T f_{2}\right\|_{L_{p}(B)} \lesssim r^{n / p} \int_{2 r}^{\infty}\|f\|_{L_{p}\left(B\left(x_{0}, t\right)\right)} \frac{d t}{t^{n / p+1}}
$$

is valid. Thus,

$$
\|T f\|_{L_{p}(B)} \lesssim\|f\|_{L_{p}(2 B)}+r^{n / p} \int_{2 r}^{\infty}\|f\|_{L_{p}\left(B\left(x_{0}, t\right)\right)} \frac{d t}{t^{n / p+1}}
$$

On the other hand,

$$
\begin{aligned}
\|f\|_{L_{p}(2 B)} & \approx r^{n / p}\|f\|_{L_{p}(2 B)} \int_{2 r}^{\infty} \frac{d t}{t^{n / p+1}} \\
& \lesssim r^{n / p} \int_{2 r}^{\infty}\|f\|_{L_{p}\left(B\left(x_{0}, t\right)\right)} \frac{d t}{t^{n / p+1}} .
\end{aligned}
$$

Thus,

$$
\|T f\|_{L_{p}(B)} \lesssim r^{n / p} \int_{2 r}^{\infty}\|f\|_{L_{p}\left(B\left(x_{0}, t\right)\right)} \frac{d t}{t^{n / p+1}}
$$

Let $p=1$. From the weak $(1,1)$ boundedness of $T$ and $(4.15)$, it follows that

$$
\begin{aligned}
\left\|T f_{1}\right\|_{W L_{1}(B)} & \leq\left\|T f_{1}\right\|_{W L_{1}\left(\mathbb{R}^{n}\right)} \lesssim\left\|f_{1}\right\|_{L_{1}\left(\mathbb{R}^{n}\right)} \\
& =\|f\|_{L_{1}(2 B)} \lesssim r^{n} \int_{2 r}^{\infty} \int_{B\left(x_{0}, t\right)}|f(y)| d y \frac{d t}{t^{n+1}} .
\end{aligned}
$$

Then, by (4.13) and (4.17), we get (4.6).

Theorem 4.5. Let $1 \leq p<\infty$, and let $\left(\varphi_{1}, \varphi_{2}\right)$ satisfy the condition

$$
\int_{r}^{\infty} \frac{\operatorname{ess} \inf _{t<s<\infty} \varphi_{1}(x, s) s^{n / p}}{t^{n / p+1}} d t \leq C \varphi_{2}(x, r),
$$

where $C$ does not depend on $x$ and $r$. Let $T$ be a sublinear operator which satisfies the condition (1.5) bounded on $L_{p}\left(\mathbb{R}^{n}\right)$ for $p>1$ and bounded from $L_{1}\left(\mathbb{R}^{n}\right)$ to $W L_{1}\left(\mathbb{R}^{n}\right)$. Then, the operator $T$ is bounded from $M_{p, \varphi_{1}}$ to $M_{p, \varphi_{2}}$ for $p>1$ and from $M_{1, \varphi_{1}}$ to $W M_{1, \varphi_{2}}$. Moreover, for $p>1$

$$
\|T f\|_{M_{p, \varphi_{2}}} \lesssim\|f\|_{M_{p, \varphi_{1}}}
$$

and for $p=1$

$$
\|T f\|_{W M_{1, \varphi_{2}}} \lesssim\|f\|_{M_{1, \varphi_{1}}}
$$


Proof. By Lemma 4.4 and Theorem 4.3, we have for $p>1$

$$
\begin{aligned}
\|T f\|_{M_{p, \varphi_{2}}} & \lesssim \sup _{x \in \mathbb{R}^{n}, r>0} \varphi_{2}(x, r)^{-1} \int_{r}^{\infty}\|f\|_{L_{p}(B(x, t))} \frac{d t}{t^{n / p+1}} \\
& \approx \sup _{x \in \mathbb{R}^{n}, r>0} \varphi_{2}(x, r)^{-1} \int_{0}^{r^{-n / p}}\|f\|_{L_{p}\left(B\left(x, t^{-p / n}\right)\right)} d t \\
& =\sup _{x \in \mathbb{R}^{n}, r>0} \varphi_{2}\left(x, r^{-p / n}\right)^{-1} \int_{0}^{r}\|f\|_{L_{p}\left(B\left(x, t^{-p / n}\right)\right)} d t \\
& \lesssim \sup _{x \in \mathbb{R}^{n}, r>0} \varphi_{1}\left(x, r^{-p / n}\right)^{-1} r\|f\|_{L_{p}(B(x, t))}=\|f\|_{M_{p, \varphi_{1}}}
\end{aligned}
$$

and for $p=1$

$$
\begin{aligned}
\|T f\|_{W M_{1, \varphi_{2}}} & \lesssim \sup _{x \in \mathbb{R}^{n}, r>0} \varphi_{2}(x, r)^{-1} \int_{r}^{\infty}\|f\|_{L_{1}(B(x, t))} \frac{d t}{t^{n+1}} \\
& \approx \sup _{x \in \mathbb{R}^{n}, r>0} \varphi_{2}(x, r)^{-1} \int_{0}^{r^{-n}}\|f\|_{L_{1}\left(B\left(x, t^{-1 / n}\right)\right)} d t \\
& =\sup _{x \in \mathbb{R}^{n}, r>0} \varphi_{2}\left(x, r^{-1 / n}\right)^{-1} \int_{0}^{r}\|f\|_{L_{1}\left(B\left(x, t^{-1 / n}\right)\right)} d t \\
& \lesssim \sup _{x \in \mathbb{R}^{n}, r>0} \varphi_{1}\left(x, r^{-1 / n}\right)^{-1} r\|f\|_{L_{1}\left(B\left(x, r^{-1 / n}\right)\right)}=\|f\|_{M_{1, \varphi_{1}}} .
\end{aligned}
$$

Corollary 4.6. Let $1 \leq p<\infty$, and $\left(\varphi_{1}, \varphi_{2}\right)$ satisfies the condition (4.18). Then, the operators $M$ and $K$ are bounded from $M_{p, \varphi_{1}}$ to $M_{p, \varphi_{2}}$ for $p>1$ and bounded from $M_{1, \varphi_{1}}$ to $W M_{1, \varphi_{2}}$.

Note that Corollary 4.6 was proved in [28].

\section{Commutators of Sublinear Operators Generated by Calderón-Zygmund Operators in the Spaces $M_{p, \varphi}$}

Let $T$ be a Calderón-Zygmund singular integral operator and $a \in \operatorname{BMO}\left(\mathbb{R}^{n}\right)$. A well-known result of Coifman et al. [29] states that the commutator operator $[a, T] f=T(a f)-a T f$ is bounded on $L_{p}\left(\mathbb{R}^{n}\right)$ for $1<p<\infty$. The commutator of Calderon-Zygmund operators plays an important role in studying the regularity of solutions of elliptic partial differential equations of second order (see, e.g., $[12,30,31])$. 
First, we introduce the definition of the space of $\mathrm{BMO}\left(\mathbb{R}^{n}\right)$.

Definition 5.1. Suppose that $f \in L_{1}^{\text {loc }}\left(\mathbb{R}^{n}\right)$, and let

$$
\|f\|_{*}=\sup _{x \in \mathbb{R}^{n}, r>0} \frac{1}{|B(x, r)|} \int_{B(x, r)}\left|f(y)-f_{B(x, r)}\right| d y<\infty,
$$

where

$$
f_{B(x, r)}=\frac{1}{|B(x, r)|} \int_{B(x, r)} f(y) d y .
$$

Define

$$
\operatorname{BMO}\left(\mathbb{R}^{n}\right)=\left\{f \in L_{1}^{\text {loc }}\left(\mathbb{R}^{n}\right):\|f\|_{*}<\infty\right\}
$$

If one regards two functions whose difference is a constant as one, then space $\operatorname{BMO}\left(\mathbb{R}^{n}\right)$ is a Banach space with respect to norm $\|\cdot\|_{*}$.

Remark 5.2. (1) The John-Nirenberg inequality: there are constants $C_{1}, C_{2}>0$ such that for all $f \in \mathrm{BMO}\left(\mathbb{R}^{n}\right)$ and $\beta>0$,

$$
\left|\left\{x \in B:\left|f(x)-f_{B}\right|>\beta\right\}\right| \leq C_{1}|B| e^{-C_{2} \beta /\|f\|_{*}}, \quad \forall B \subset \mathbb{R}^{n} .
$$

(2) The John-Nirenberg inequality implies that

$$
\|f\|_{*} \approx \sup _{x \in \mathbb{R}^{n}, r>0}\left(\frac{1}{|B(x, r)|} \int_{B(x, r)}\left|f(y)-f_{B(x, r)}\right|^{p} d y\right)^{1 / p}
$$

for $1<p<\infty$.

(3) Let $f \in \operatorname{BMO}\left(\mathbb{R}^{n}\right)$. Then, there is a constant $C>0$ such that

$$
\left|f_{B(x, r)}-f_{B(x, t)}\right| \leq C\|f\|_{*} \ln \frac{t}{r} \quad \text { for } 0<2 r<t
$$

where $C$ is independent of $f, x, r$, and $t$.

In [24], the following statement was proved for the commutators of sublinear operators, containing the result in $[18,19]$.

Theorem 5.3. Let $1<p<\infty, \varphi(x, r)$ which satisfies the conditions (3.4) and (3.5) and $a \in$ $B M O\left(\mathbb{R}^{n}\right)$. Suppose that $T$ is a linear operator and satisfies the condition (1.5). If the operator $[a, T]$ is bounded on $L_{p}\left(\mathbb{R}^{n}\right)$, then the operator $[a, T]$ is bounded on $M_{p, \varphi}$.

Remark 5.4. Note that Theorem 5.3 in the following form is also valid. Let $1<p<\infty, \varphi(x, r)$ satisfy the conditions (3.4) and (3.5) and $a \in \mathrm{BMO}\left(\mathbb{R}^{n}\right)$. Suppose that $T_{a}$ is a sublinear 
operator satisfies the condition (1.6) and bounded on $L_{p}\left(\mathbb{R}^{n}\right)$, then the operator $T_{a}$ is bounded on $M_{p, \varphi}$.

Lemma 5.5. Let $1<p<\infty, a \in B M O\left(\mathbb{R}^{n}\right)$, and a sublinear operator $T_{a}$ satisfies the condition (1.6) and bounded on $L_{p}\left(\mathbb{R}^{n}\right)$.

Then,

$$
\left\|T_{a} f\right\|_{L_{p}(B)} \lesssim\|a\|_{*} r^{n / p} \int_{2 r}^{\infty}\left(1+\ln \frac{t}{r}\right) t^{-(n / p)-1}\|f\|_{L_{p}\left(B\left(x_{0}, t\right)\right)} d t
$$

holds for any ball $B=B\left(x_{0}, r\right)$ and for all $f \in L_{p}^{\text {loc }}\left(\mathbb{R}^{n}\right)$.

Proof. $1<p<\infty, a \in \mathrm{BMO}\left(\mathbb{R}^{n}\right)$, and a sublinear operator $T_{a}$ satisfies the condition (1.6). For arbitrary $x_{0} \in \mathbb{R}^{n}$, set $B=B\left(x_{0}, r\right)$ for the ball centered at $x_{0}$ and of radius $r$. Write $f=f_{1}+f_{2}$ with $f_{1}=f X_{2 B}$ and $f_{2}=f X^{c}(2 B)$. Hence,

$$
\left\|T_{a} f\right\|_{L_{p}(B)} \leq\left\|T_{a} f_{1}\right\|_{L_{p}(B)}+\left\|T_{a} f_{2}\right\|_{L_{p}(B)} .
$$

From the boundedness of $T_{a}$ in $L_{p}\left(\mathbb{R}^{n}\right)$, it follows that

$$
\begin{aligned}
\left\|T_{a} f_{1}\right\|_{L_{p}(B)} & \leq\left\|T_{a} f_{1}\right\|_{L_{p}\left(\mathbb{R}^{n}\right)} \\
& \lesssim\|a\|_{*}\left\|f_{1}\right\|_{L_{p}\left(\mathbb{R}^{n}\right)}=\|a\|_{*}\|f\|_{L_{p}(2 B)}
\end{aligned}
$$

For $x \in B$, we have

$$
\begin{aligned}
\left|T_{a} f_{2}(x)\right| & \lesssim \int_{\mathbb{R}^{n}} \frac{|a(y)-a(x)|}{|x-y|^{n}}\left|f_{2}(y)\right| d y \\
& \approx \int_{{ }^{(}(2 B)} \frac{|a(y)-a(x)|}{\left|x_{0}-y\right|^{n}}|f(y)| d y .
\end{aligned}
$$

Then,

$$
\begin{aligned}
\left\|T_{a} f_{2}\right\|_{L_{p}(B)} & \lesssim\left(\int_{B}\left(\int_{{ }^{c}(2 B)} \frac{|a(y)-a(x)|}{\left|x_{0}-y\right|^{n}}|f(y)| d y\right)^{p} d x\right)^{1 / p} \\
& \lesssim\left(\int_{B}\left(\int_{{ }^{c}(2 B)} \frac{\left|a(y)-a_{B}\right|}{\left|x_{0}-y\right|^{n}}|f(y)| d y\right)^{p} d x\right)^{1 / p} \\
& +\left(\int_{B}\left(\int_{{ }^{c}(2 B)} \frac{\left|a(x)-a_{B}\right|}{\left|x_{0}-y\right|^{n}}|f(y)| d y\right)^{p} d x\right)^{1 / p} \\
& =I_{1}+I_{2} .
\end{aligned}
$$


Let us estimate $I_{1}$

$$
\begin{aligned}
I_{1} & \approx r^{n / p} \int_{{ }^{c}(2 B)} \frac{\left|a(y)-a_{B}\right|}{\left|x_{0}-y\right|^{n}}|f(y)| d y \\
& \approx r^{n / p} \int_{{ }^{\prime}(2 B)}\left|a(y)-a_{B}\right||f(y)| \int_{\left|x_{0}-y\right|}^{\infty} \frac{d t}{t^{n+1}} d y \\
& \approx r^{n / p} \int_{2 r}^{\infty} \int_{2 r \leq\left|x_{0}-y\right| \leq t}\left|a(y)-a_{B}\right||f(y)| d y \frac{d t}{t^{n+1}} \\
& \lesssim r^{n / p} \int_{2 r}^{\infty} \int_{B\left(x_{0}, t\right)}\left|a(y)-a_{B}\right||f(y)| d y \frac{d t}{t^{n+1}} .
\end{aligned}
$$

Applying Hölder's inequality and by (5.5), (5.6), we get

$$
\begin{aligned}
I_{1} \lesssim & r^{n / p} \int_{2 r}^{\infty} \int_{B\left(x_{0}, t\right)}\left|a(y)-a_{B\left(x_{0}, t\right)}\right||f(y)| d y \frac{d t}{t^{n+1}} \\
& +r^{n / p} \int_{2 r}^{\infty}\left|a_{B\left(x_{0}, r\right)}-a_{B\left(x_{0}, t\right)}\right| \int_{B\left(x_{0}, t\right)}|f(y)| d y \frac{d t}{t^{n+1}} \\
\lesssim & r^{n / p} \int_{2 r}^{\infty}\left(\int_{B\left(x_{0}, t\right)}\left|a(y)-a_{B\left(x_{0}, t\right)}\right|^{p^{\prime}} d y\right)^{1 / p^{\prime}}\|f\|_{L_{p}\left(B\left(x_{0}, t\right)\right)} \frac{d t}{t^{n+1}} \\
& +r^{n / p} \int_{2 r}^{\infty}\left|a_{B\left(x_{0}, r\right)}-a_{B\left(x_{0}, t\right)}\right|\|f\|_{L_{p}\left(B\left(x_{0}, t\right)\right)} \frac{d t}{t^{n / p+1}} \\
\lesssim & \|a\|_{*} r^{n / p} \int_{2 r}^{\infty}\left(1+\ln \frac{t}{r}\right)\|f\|_{L_{p}\left(B\left(x_{0}, t\right)\right)} \frac{d t}{t^{n / p+1}} .
\end{aligned}
$$

In order to estimate $I_{2}$, note that

$$
I_{2}=\left(\int_{B}\left|a(x)-a_{B}\right|^{p} d x\right)^{1 / p} \int_{{ }^{c}(2 B)} \frac{|f(y)|}{\left|x_{0}-y\right|^{n}} d y .
$$

By (5.5), we get

$$
I_{2} \lesssim\|a\|_{*} r^{n / p} \int_{{ }^{c}(2 B)} \frac{|f(y)|}{\left|x_{0}-y\right|^{n}} d y
$$

Thus, by (4.12),

$$
I_{2} \lesssim\|a\|_{*} r^{n / p} \int_{2 r}^{\infty}\|f\|_{L_{p}\left(B\left(x_{0}, t\right)\right)} \frac{d t}{t^{n / p+1}} .
$$


Summing up $I_{1}$ and $I_{2}$, for all $p \in[1, \infty)$, we get

$$
\left\|T_{a} f_{2}\right\|_{L_{p}(B)} \lesssim\|a\|_{*} r^{n / p} \int_{2 r}^{\infty}\left(1+\ln \frac{t}{r}\right)\|f\|_{L_{p}\left(B\left(x_{0}, t\right)\right)} \frac{d t}{t^{n / p+1}} .
$$

Finally,

$$
\left\|T_{a} f\right\|_{L_{p}(B)} \lesssim\|a\|_{*}\|f\|_{L_{p}(2 B)}+\|a\|_{*} r^{n / p} \int_{2 r}^{\infty}\left(1+\ln \frac{t}{r}\right)\|f\|_{L_{p}\left(B\left(x_{0}, t\right)\right)} \frac{d t}{t^{n / p+1}}
$$

and statement of Lemma 5.5 follows by (4.15).

The following theorem is true.

Theorem 5.6. Let $1<p<\infty, a \in B M O\left(\mathbb{R}^{n}\right)$ and $\left(\varphi_{1}, \varphi_{2}\right)$ satisfy the condition

$$
\int_{r}^{\infty}\left(1+\ln \frac{t}{r}\right) \frac{\operatorname{ess} \inf _{t<s<\infty} \varphi_{1}(x, s) s^{n / p}}{t^{n / p+1}} d t \leq C \varphi_{2}(x, r),
$$

where $C$ does not depend on $x$ and $r$. Suppose that $T_{a}$ is a sublinear operator which satisfies the condition (1.6) and bounded on $L_{p}\left(\mathbb{R}^{n}\right)$.

Then, the operator $T_{a}$ is bounded from $M_{p, \varphi_{1}}$ to $M_{p, \varphi_{2}}$. Moreover,

$$
\left\|T_{a} f\right\|_{M_{p, \psi_{2}}} \lesssim\|a\|_{*}\|f\|_{M_{p, \varphi_{1}}} .
$$

Proof. The statement of Theorem 5.6 is followed by Lemma 5.5 and Theorem 4.3 in the same manner as in the proof of Theorem 4.5.

For the sublinear commutator of the maximal operator

$$
M_{a}(f)(x)=\sup _{t>0}|B(x, t)|^{-1} \int_{B(x, t)}|a(x)-a(y)||f(y)| d y
$$

and for the linear commutator of the Calderón-Zygmund operator $[a, K]$ from Theorem 5.6, we get the following new results.

Corollary 5.7. Let $1<p<\infty,\left(\varphi_{1}, \varphi_{2}\right)$ satisfy the condition (5.19) and $a \in B M O\left(\mathbb{R}^{n}\right)$. Then, the sublinear commutator operator $M_{a}$ is bounded from $M_{p, \varphi_{1}}$ to $M_{p, \varphi_{2}}$.

Corollary 5.8. Let $1<p<\infty,\left(\varphi_{1}, \varphi_{2}\right)$ satisfy the condition (5.19) and $a \in B M O\left(\mathbb{R}^{n}\right)$. Then, Calderón-Zygmund singular integral $K f(x)$ exists for a.e. $x \in \mathbb{R}^{n}$ and the operator $[a, K]$ is bounded from $M_{p, \varphi_{1}}$ to $M_{p, \varphi_{2}}$.

Note that when the conditions of Corollary 5.8 are satisfied, the existence of $K f(x)$ for a.e. $x \in \mathbb{R}^{n}$ was proved in [28]. 


\section{Some Applications}

In this section, we will apply Theorems 4.5 and 5.6 to several particular operators such as the pseudodifferential operators, Littlewood-Paley operator, Marcinkiewicz operator, and Bochner-Riesz operator.

\subsection{Pseudodifferential Operators}

Pseudodifferential operators are generalizations of differential operators and singular integrals. Let $m$ be real number, $0 \leq \delta<1$ and $0 \leq \rho<1$. Following [32, 33], a symbol in $S_{\rho, \delta}^{m}$ is a smooth function $\sigma(x, \xi)$ defined on $\mathbb{R}^{n} \times \mathbb{R}^{n}$ such that for all multi-indices $\alpha$ and $\beta$ the following estimate holds:

$$
\left|D_{x}^{\alpha} D_{\xi}^{\beta} \sigma(x, \xi)\right| \leq C_{\alpha \beta}(1+|\xi|)^{m-\rho|\beta|+\delta|\alpha|},
$$

where $C_{\alpha \beta}>0$ is independent of $x$ and $\xi$. A symbol in $S_{\rho, \delta}^{-\infty}$ is one which satisfies the above estimates for each real number $m$.

The operator $A$ given by

$$
A f(x)=\int_{\mathbb{R}^{n}} \sigma(x, \xi) e^{2 \pi i x \xi} \widehat{f}(\xi) d \xi
$$

is called a pseudodifferential operator with symbol $\sigma(x, \xi) \in S_{\rho, \delta}^{m}$, where $f$ is a Schwartz function and $\widehat{f}$ denotes the Fourier transform of $f$. As usual, $L_{\rho, \delta}^{m}$ will denote the class of pseudodifferential operators with symbols in $S_{\rho, \delta}^{m}$.

Miller [34] showed the boundedness of $L_{1,0}^{0}$ pseudodifferential operators on weighted $L_{p}(1<p<\infty)$ spaces whenever the weight function belongs to Muckenhoupt's class $A_{p}$. In [1], it is shown that pseudodifferential operators in $L_{1,0}^{0}$ are Calderón-Zygmund operators, then from Corollary 5.8, we get the following new results.

Corollary 6.1. Let $1 \leq p<\infty$, and let $\left(\varphi_{1}, \varphi_{2}\right)$ satisfy the condition (4.18). If $A$ is a pseudodifferential operator of the Hörmander class $L_{1,0}^{0}$, then the operator $A$ is bounded from $M_{p, \varphi_{1}}$ to $M_{p, \varphi_{2}}$ for $p>1$ and bounded from $M_{1, \varphi_{1}}$ to $W M_{1, \varphi_{2}}$.

Corollary 6.2. Let $1<p<\infty,\left(\varphi_{1}, \varphi_{2}\right)$ satisfy the condition (5.19) and $a \in B M O\left(\mathbb{R}^{n}\right)$. Let also $A$ be a pseudodifferential operator of the Hörmander class $L_{1,0}^{0}$. Then, the commutator operator $[a, A]$ is bounded from $M_{p, \varphi_{1}}$ to $M_{p, \varphi_{2}}$.

\subsection{Littlewood-Paley Operator}

The Littlewood-Paley functions play an important role in classical harmonic analysis, for example, in the study of nontangential convergence of Fatou type and boundedness of Riesz transforms and multipliers $[4-6,35]$. The Littlewood-Paley operator (see $[6,36])$ is defined as follows. 
Definition 6.3. Suppose that $\psi \in L_{1}\left(\mathbb{R}^{n}\right)$ satisfies

$$
\int_{\mathbb{R}^{n}} \psi(x) d x=0
$$

Then, the generalized Littlewood-Paley $g$ function $g_{\psi}$ is defined by

$$
g_{\psi}(f)(x)=\left(\int_{0}^{\infty}\left|F_{t}(f)(x)\right|^{2} \frac{d t}{t}\right)^{1 / 2},
$$

where $\psi_{t}(x)=t^{-n} \psi(x / t)$ for $t>0$ and $F_{t}(f)=\psi_{t} * f$.

The sublinear commutator of the operator $g_{\psi}$ is defined by

$$
\left[a, g_{\psi}\right](f)(x)=\left(\int_{0}^{\infty}\left|F_{t}^{a}(f)(x)\right|^{2} \frac{d t}{t}\right)^{1 / 2}
$$

where

$$
F_{t}^{a}(f)(x)=\int_{\mathbb{R}^{n}}[a(x)-a(y)] \psi_{t}(x-y) f(y) d y .
$$

The following theorem is valid (see [3, Theorem 5.1.2]).

Theorem 6.4. Suppose that $\psi \in L_{1}\left(\mathbb{R}^{n}\right)$ satisfies (6.3) and the following properties:

$$
\begin{gathered}
|\psi(x)| \leq \frac{C}{(1+|x|)^{n+\alpha}}, \quad x \in \mathbb{R}^{n}, \\
\int_{\mathbb{R}^{n}}|\psi(x+h)-\psi(x)| d x \leq C|h|^{\alpha}, \quad h \in \mathbb{R}^{n},
\end{gathered}
$$

where $C$ and $\alpha>0$ are both independent of $x$ and $h$. Then, $g_{\psi}$ is bounded on $L_{p}\left(\mathbb{R}^{n}\right)$ for all $1<p<\infty$, and bounded from $L_{1}\left(\mathbb{R}^{n}\right)$ to $W L_{1}\left(\mathbb{R}^{n}\right)$.

Let $H$ be the space $H=\left\{h:\|h\|=\left(\int_{0}^{\infty}|h(t)|^{2} d t / t\right)^{1 / 2}<\infty\right\}$, then for each fixed $x \in \mathbb{R}^{n}, F_{t}(f)(x)$ may be viewed as a mapping from $[0, \infty)$ to $H$, and it is clear that $g_{\psi}(f)(x)=$ $\left\|F_{t}(f)(x)\right\|$. In fact, by Minkowski inequality and the conditions on $\psi$, we get

$$
\begin{aligned}
g_{\psi}(f)(x) & \leq \int_{\mathbb{R}^{n}}|f(y)|\left(\int_{0}^{\infty}\left|\psi_{t}(x-y)\right|^{2} \frac{d t}{t}\right)^{1 / 2} d y \\
& \leq C \int_{\mathbb{R}^{n}}|f(y)|\left(\int_{0}^{\infty} \frac{t^{-2 n}}{(1+|x-y| / t)^{2(n+1)}} \frac{d t}{t}\right)^{1 / 2} d y \\
& =C \int_{\mathbb{R}^{n}} \frac{|f(y)|}{|x-y|^{n}} d y .
\end{aligned}
$$

Thus, we get the following. 
Corollary 6.5. Let $1 \leq p<\infty,\left(\varphi_{1}, \varphi_{2}\right)$ satisfies the condition (4.18) and $\psi \in L_{1}\left(\mathbb{R}^{n}\right)$ satisfies (6.3) and (6.7). Then the operator $g_{\psi}$ is bounded from $M_{p, \varphi_{1}}$ to $M_{p, \varphi_{2}}$ for $p>1$ and bounded from $M_{1, \varphi_{1}}$ to $W M_{1, \varphi_{2}}$.

Corollary 6.6. Let $1<p<\infty,\left(\varphi_{1}, \varphi_{2}\right)$ satisfies the condition (5.19), $a \in B M O\left(\mathbb{R}^{n}\right)$ and $\psi \in L_{1}\left(\mathbb{R}^{n}\right)$ satisfies (6.3) and (6.7). Then the operator $\left[a, g_{\psi}\right]$ is bounded from $M_{p, \varphi_{1}}$ to $M_{p, \varphi_{2}}$.

\subsection{Marcinkiewicz Operator}

Let $S^{n-1}=\left\{x \in \mathbb{R}^{n}:|x|=1\right\}$ be the unit sphere in $\mathbb{R}^{n}$ equipped with the Lebesgue measure $d \sigma$. Suppose that $\Omega$ satisfies the following conditions.

(a) $\Omega$ is the homogeneous function of degree zero on $\mathbb{R}^{n} \backslash\{0\}$; that is,

$$
\Omega(t x)=\Omega(x), \quad \text { for any } t>0, x \in \mathbb{R}^{n} \backslash\{0\} .
$$

(b) $\Omega$ has mean zero on $S^{n-1}$; that is,

$$
\int_{S^{n-1}} \Omega\left(x^{\prime}\right) d \sigma\left(x^{\prime}\right)=0
$$

(c) $\Omega \in \operatorname{Lip}_{\gamma}\left(S^{n-1}\right), 0<\gamma \leq 1$, that is there exists a constant $M>0$ such that

$$
\left|\Omega\left(x^{\prime}\right)-\Omega\left(y^{\prime}\right)\right| \leq M\left|x^{\prime}-y^{\prime}\right|^{\gamma} \text { for any } x^{\prime}, y^{\prime} \in S^{n-1} \text {. }
$$

In 1958, Stein [35] defined the Marcinkiewicz integral of higher dimension $\mu_{\Omega}$ as

$$
\mu_{\Omega}(f)(x)=\left(\int_{0}^{\infty}\left|F_{\Omega, t}(f)(x)\right|^{2} \frac{d t}{t^{3}}\right)^{1 / 2}
$$

where

$$
F_{\Omega, t}(f)(x)=\int_{|x-y| \leq t} \frac{\Omega(x-y)}{|x-y|^{n-1}} f(y) d y
$$

Since Stein's work in 1958, the continuity of Marcinkiewicz integral has been extensively studied as a research topic and also provides useful tools in harmonic analysis [3-6].

The sublinear commutator of the operator $\mu_{\Omega}$ is defined by

$$
\left[a, \mu_{\Omega}\right](f)(x)=\left(\int_{0}^{\infty}\left|F_{\Omega, t, a}(f)(x)\right|^{2} \frac{d t}{t^{3}}\right)^{1 / 2},
$$


where

$$
F_{\Omega, t, a}(f)(x)=\int_{|x-y| \leq t} \frac{\Omega(x-y)}{|x-y|^{n-1}}[a(x)-a(y)] f(y) d y .
$$

Let $H$ be the space $H=\left\{h:\|h\|=\left(\int_{0}^{\infty}|h(t)|^{2} d t / t^{3}\right)^{1 / 2}<\infty\right\}$. Then, it is clear that $\mu_{\Omega}(f)(x)=\left\|F_{\Omega, t}(f)(x)\right\|$.

By Minkowski inequality and the conditions on $\Omega$, we get

$$
\mu_{\Omega}(f)(x) \leq \int_{\mathbb{R}^{n}} \frac{|\Omega(x-y)|}{|x-y|^{n-1}}|f(y)|\left(\int_{|x-y|}^{\infty} \frac{d t}{t^{3}}\right)^{1 / 2} d y \leq C \int_{\mathbb{R}^{n}} \frac{|f(y)|}{|x-y|^{n}} d y
$$

Thus, $\mu_{\Omega}$ satisfies the condition (1.5). It is known that $\mu_{\Omega}$ is bounded on $L_{p}\left(\mathbb{R}^{n}\right)$ for $p>1$ and bounded from $L_{1}\left(\mathbb{R}^{n}\right)$ to $W L_{1}\left(\mathbb{R}^{n}\right)$ (see [37]), then from Theorems 4.5 and 5.6, we get the following collory.

Corollary 6.7. Let $1 \leq p<\infty$ and $\left(\varphi_{1}, \varphi_{2}\right)$ satisfy the condition (4.18), and let $\Omega$ satisfy the conditions $(a)-(c)$. Then, $\mu_{\Omega}$ is bounded from $M_{p, \varphi_{1}}$ to $M_{p, \varphi_{2}}$ for $p>1$ and bounded from $M_{1, \varphi_{1}}$ to $W M_{1, \varphi_{2}}$.

Corollary 6.8. Let $1<p<\infty,\left(\varphi_{1}, \varphi_{2}\right)$ satisfy the condition (5.19), $a \in B M O\left(\mathbb{R}^{n}\right)$, and $\Omega$ satisfy the conditions (a)-(c). Then, $\left[a, \mu_{\Omega}\right]$ is bounded from $M_{p, \varphi_{1}}$ to $M_{p, \varphi_{2}}$.

\subsection{Bochner-Riesz Operator}

Let $\delta>(n-1) / 2, B_{t}^{\delta}(\widehat{f})(\xi)=\left(1-t^{2}|\xi|^{2}\right)_{+}^{\delta} \widehat{f}(\xi)$ and $B_{t}^{\delta}(x)=t^{-n} B^{\delta}(x / t)$ for $t>0$. The maximal Bochner-Riesz operator is defined by (see $[38,39])$

$$
B_{*}^{\delta}(f)(x)=\sup _{t>0}\left|B_{t}^{\delta}(f)(x)\right|
$$

Let $H$ be the space $H=\left\{h:\|h\|=\sup _{t>0}|h(t)|<\infty\right\}$, then it is clear that $B_{*}^{\delta}(f)(x)=$ $\left\|B_{t}^{\delta}(f)(x)\right\|$.

By the condition on $B_{r}^{\delta}$ (see [2]), we have

$$
\begin{aligned}
\left|B_{r}^{\delta}(x-y)\right| & \leq C r^{-n}(1+|x-y| / r)^{-(\delta+(n+1) / 2)} \\
& =C\left(\frac{r}{r+|x-y|}\right)^{\delta-(n-1) / 2} \frac{1}{(r+|x-y|)^{n}} \\
& \leq|x-y|^{-n}, \\
B_{*}^{\delta}(f)(x) & \leq C \int_{\mathbb{R}^{n}} \frac{|f(y)|}{|x-y|^{n}} d y .
\end{aligned}
$$


Thus, $B_{*}^{\delta}$ satisfies the condition (1.5). It is known that $B_{*}^{\delta}$ is bounded on $L_{p}\left(\mathbb{R}^{n}\right)$ for $p>1$, and bounded from $L_{1}\left(\mathbb{R}^{n}\right)$ to $W L_{1}\left(\mathbb{R}^{n}\right)$, then from Theorems 4.5 and 5.6, we get the following corollary.

Corollary 6.9. Let $1 \leq p<\infty,\left(\varphi_{1}, \varphi_{2}\right)$ satisfy the condition (4.18) and $\delta>(n-1) / 2$. Then, the operator $B_{*}^{\delta}$ is bounded from $M_{p, \varphi_{1}}$ to $M_{p, \varphi_{2}}$ for $p>1$ and bounded from $M_{1, \varphi_{1}}$ to $W M_{1, \varphi_{2}}$.

Corollary 6.10. Let $1<p<\infty,\left(\varphi_{1}, \varphi_{2}\right)$ satisfy the condition (5.19), $\delta>(n-1) / 2$ and $a \in$ $B M O\left(\mathbb{R}^{n}\right)$. Then, the operator $\left[a, B_{t}^{\delta}\right]$ is bounded from $M_{p, \varphi_{1}}$ to $M_{p, \varphi_{2}}$.

Remark 6.11. Recall that under the assumption that $\varphi(x, r)$ satisfies the conditions (3.4) and (3.5), the Corollaries 6.9 and 6.10 were proved in [38].

\section{Acknowledgments}

The authors express their thank to prof A. Serbetci for helpful comments on the paper of this paper. The authors would like to express their gratitude to the referees for his very valuable comments and suggestions. The research of V. Guliyev was partially supported by the grant of Science Development Foundation under the President of the Republic of Azerbaijan project no-01/023. The research of V. Guliyev and T. Karaman was partially supported by the grant of 2010-Ahi Evran University Scientific Research Projects (BAP FBA-10-05) and by the Scientific and Technological Research Council of Turkey (TUBITAK Project no: 110T695).

\section{References}

[1] R. R. Coifman and Y. Meyer, Au Delà des Opérateurs Pseudo-Différentiels, vol. 57 of Astérisque, Société Mathématique de France, Paris, France, 1978.

[2] J. García-Cuerva and J. L. Rubio de Francia, Weighted Norm Inequalities and Related Topics, vol. 116 of North-Holland Mathematics Studies, North-Holland, Amsterdam, The Netherlands, 1985.

[3] S. Lu, Y. Ding, and D. Yan, Singular Integrals and Related Topics, World Scientific Publishing, Hackensack, NJ, USA, 2007.

[4] E. M. Stein, Singular Integrals and Differentiability Properties of Functions, Princeton Mathematical Series, No. 30, Princeton University Press, Princeton, NJ, USA, 1970.

[5] E. M. Stein, Harmonic Analysis: Real-Variable Methods, Orthogonality, and Oscillatory Integrals, vol. 43 of Princeton Mathematical Series, Princeton University Press, Princeton, NJ, USA, 1993.

[6] A. Torchinsky, Real-Variable Methods in Harmonic Analysis, vol. 123 of Pure and Applied Mathematics, Academic Press, Orlando, Fla, USA, 1986.

[7] F. Soria and G. Weiss, "A remark on singular integrals and power weights," Indiana University Mathematics Journal, vol. 43, no. 1, pp. 187-204, 1994.

[8] G. Lu, S. Lu, and D. Yang, "Singular integrals and commutators on homogeneous groups," Analysis Mathematica, vol. 28, no. 2, pp. 103-134, 2002.

[9] C. B. Morrey, Jr., "On the solutions of quasi-linear elliptic partial differential equations," Transactions of the American Mathematical Society, vol. 43, no. 1, pp. 126-166, 1938.

[10] J. Peetre, "On the theory of $M_{p, \lambda}$," The Journal of Functional Analysis, vol. 4, pp. 71-87, 1969.

[11] F. Chiarenza and M. Frasca, "Morrey spaces and Hardy-Littlewood maximal function," Rendiconti di Matematica e delle sue Applicazioni. Serie VII, vol. 7, no. 3-4, pp. 273-279, 1987.

[12] G. Di Fazio and M. A. Ragusa, "Interior estimates in Morrey spaces for strong solutions to nondivergence form equations with discontinuous coefficients," Journal of Functional Analysis, vol. 112, no. 2, pp. 241-256, 1993.

[13] V. S. Guliyev, Integral operators on function spaces on the homogeneous groups and on domains in $R n$, Doctor's Degree Dissertation, Mathematical Institute, Moscow, Russia, 1994.

[14] V. S. Guliyev, Function Spaces, Integral Operators and Two Weighted Inequalities on Homogeneous Groups, Some Applications, Baku, Azerbaijan, 1999. 
[15] V.S. Guliyev, "Boundedness of the maximal, potential and singular operators in the generalized Morrey spaces," Journal of Inequalities and Applications, vol. 2009, Article ID 503948, 20 pages, 2009.

[16] V. S. Guliyev, J. J. Hasanov, and S. G. Samko, "Boundedness of the maximal, potential and singular operators in the generalized variable exponent Morrey spaces," Mathematica Scandinavica, vol. 107, no. 2, pp. 285-304, 2010.

[17] Y. Lin, "Strongly singular Calderón-Zygmund operator and commutator on Morrey type spaces," Acta Mathematica Sinica (English Series), vol. 23, no. 11, pp. 2097-2110, 2007.

[18] T. Mizuhara, "Boundedness of some classical operators on generalized Morrey spaces," in Harmonic Analysis (Sendai, 1990), S. Igari, Ed., ICM 90 Satellite Conference Proceedings, pp. 183-189, Springer, Tokyo, Japan, 1991.

[19] E. Nakai, "Hardy-Littlewood maximal operator, singular integral operators and the Riesz potentials on generalized Morrey spaces," Mathematische Nachrichten, vol. 166, pp. 95-103, 1994.

[20] V. I. Burenkov, V. S. Guliev, and G. V. Guliev, "Necessary and sufficient conditions for the boundedness of the fractional maximal operator in local Morrey-type spaces," Doklady Akademii Nauk, vol. 409, no. 4, pp. 443-447, 2006.

[21] V. I. Burenkov, H. V. Guliyev, and V. S. Guliyev, "Necessary and sufficient conditions for the boundedness of fractional maximal operators in local Morrey-type spaces," Journal of Computational and Applied Mathematics, vol. 208, no. 1, pp. 280-301, 2007.

[22] V. I. Burenkov, V. S. Guliyev, A. Serbetci, and T. V. Tararykova, "Necessary and sufficient conditions for the boundedness of genuine singular integral operators in local Morrey-type spaces," Eurasian Mathematical Journal, vol. 1, no. 1, pp. 32-53, 2010.

[23] V. I. Burenkov, A. Gogatishvili, V. S. Guliyev, and R. Ch. Mustafayev, "Boundedness of the fractional maximal operator in local Morrey-type spaces," Complex Variables and Elliptic Equations. An International Journal of Elliptic Equations and Complex Analysis, vol. 55, no. 8-10, pp. 739-758, 2010.

[24] Y. Ding, D. Yang, and Z. Zhou, "Boundedness of sublinear operators and commutators on $L^{p, w}\left(\mathbb{R}^{n}\right)$," Yokohama Mathematical Journal, vol. 46, no. 1, pp. 15-27, 1998.

[25] D. Fan, S. Lu, and D. Yang, "Boundedness of operators in Morrey spaces on homogeneous spaces and its applications," Acta Mathematica Sinica. New Series, vol. 14, supplement, pp. 625-634, 1998.

[26] S. Lu, D. Yang, and Z. Zhou, "Sublinear operators with rough kernel on generalized Morrey spaces," Hokkaido Mathematical Journal, vol. 27, no. 1, pp. 219-232, 1998.

[27] M. Carro, L. Pick, J. Soria, and V. D. Stepanov, "On embeddings between classical Lorentz spaces," Mathematical Inequalities \& Applications, vol. 4, no. 3, pp. 397-428, 2001.

[28] A. Akbulut, V. S. Guliyev, and R. Mustafayev, "Boundedness of the maximal operator and singular integral operator in generalized Morrey spaces," Preprint, Institute of Mathematics, AS CR, Prague, 2010.

[29] R. R. Coifman, R. Rochberg, and G. Weiss, "Factorization theorems for Hardy spaces in several variables," Annals of Mathematics. Second Series, vol. 103, no. 3, pp. 611-635, 1976.

[30] F. Chiarenza, M. Frasca, and P. Longo, "Interior $W^{2, p}$-estimates for nondivergence elliptic equations with discontinuous coefficients," Ricerche di Matematica, vol. 40, no. 1, pp. 149-168, 1991.

[31] F. Chiarenza, M. Frasca, and P. Longo, " $W^{2, p}$-solvability of the Dirichlet problem for nondivergence elliptic equations with VMO coefficients," Transactions of the American Mathematical Society, vol. 336, no. 2, pp. 841-853, 1993.

[32] L. Hörmander, "Pseudo-differential operators and hypo-elliptic operators," in Proceedings of the Symposium in Pure Mathematics of the American Mathematical Society, pp. 138-183, 1967.

[33] M. E. Taylor, Pseudo-Differential Operators and Nonlinear PDE, vol. 100 of Progress in Mathematics, Birkhäuser, Boston, Mass, USA, 1991.

[34] N. Miller, "Weighted Sobolev spaces and pseudodifferential operators with smooth symbols," Transactions of the American Mathematical Society, vol. 269, no. 1, pp. 91-109, 1982.

[35] E. M. Stein, “On the functions of Littlewood-Paley, Lusin, and Marcinkiewicz," Transactions of the American Mathematical Society, vol. 88, pp. 430-466, 1958.

[36] L. Liu, "Weighted weak type estimates for commutators of Littlewood-Paley operator," Japanese Journal of Mathematics. New Series, vol. 29, no. 1, pp. 1-13, 2003.

[37] A. Torchinsky and S. L. Wang, "A note on the Marcinkiewicz integral," Colloquium Mathematicum, vol. 60/61, no. 1, pp. 235-243, 1990.

[38] Y. Liu and D. Chen, "The boundedness of maximal Bochner-Riesz operator and maximal commutator on Morrey type spaces," Analysis in Theory and Applications, vol. 24, no. 4, pp. 321-329, 2008.

[39] L. Lanzhe and L. Shanzhen, "Weighted weak type inequalities for maximal commutators of BochnerRiesz operator," Hokkaido Mathematical Journal, vol. 32, no. 1, pp. 85-99, 2003. 


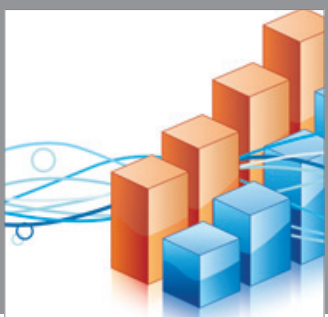

Advances in

Operations Research

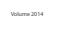

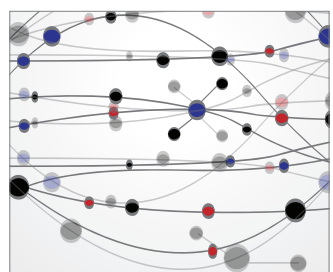

\section{The Scientific} World Journal
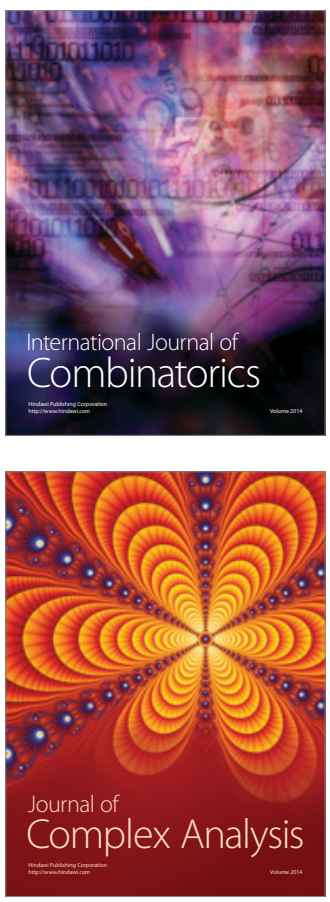

International Journal of

Mathematics and

Mathematical

Sciences
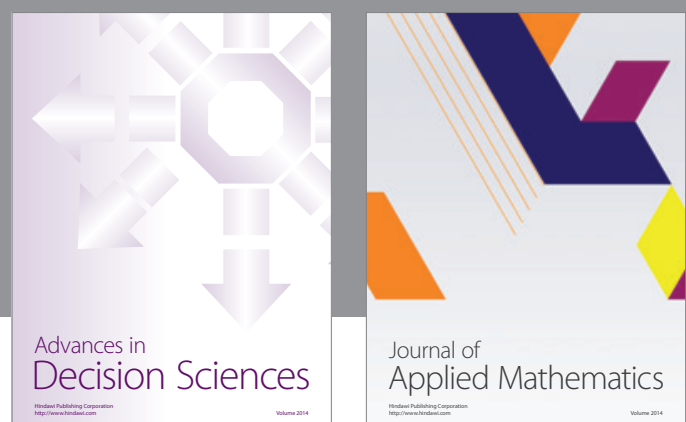

Journal of

Applied Mathematics
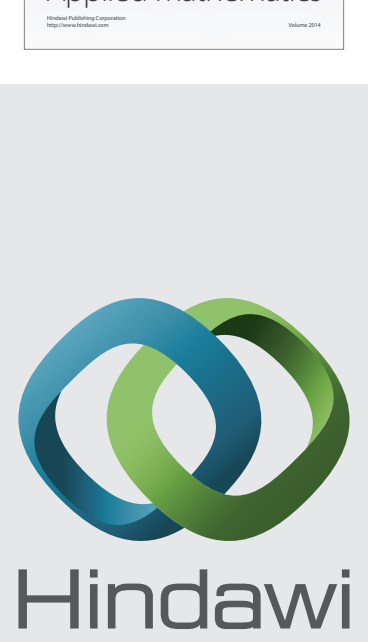

Submit your manuscripts at http://www.hindawi.com
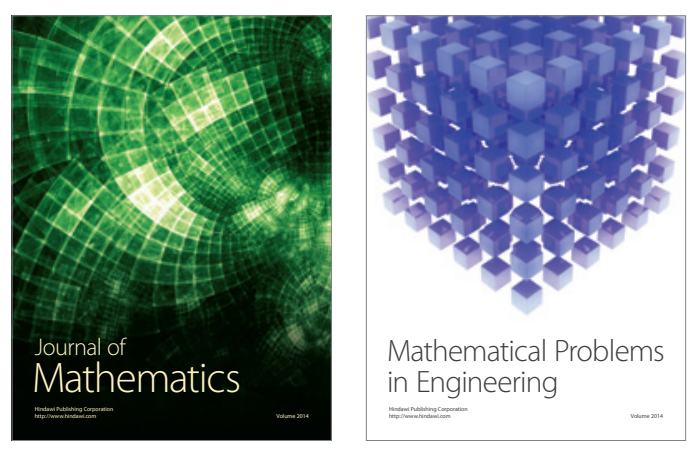

Mathematical Problems in Engineering
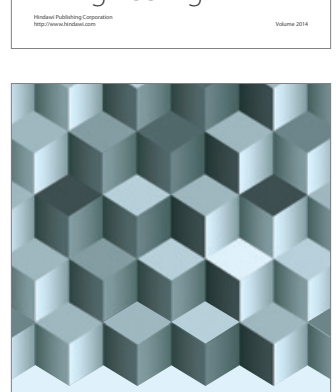

Journal of

Function Spaces
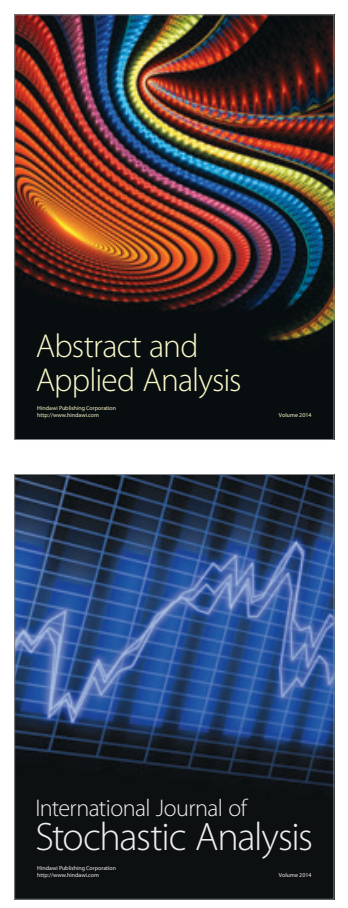

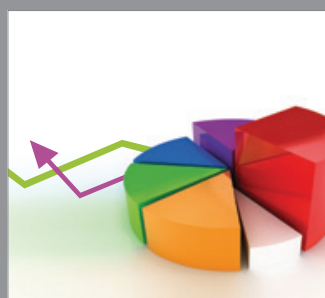

ournal of

Probability and Statistics

Promensencen
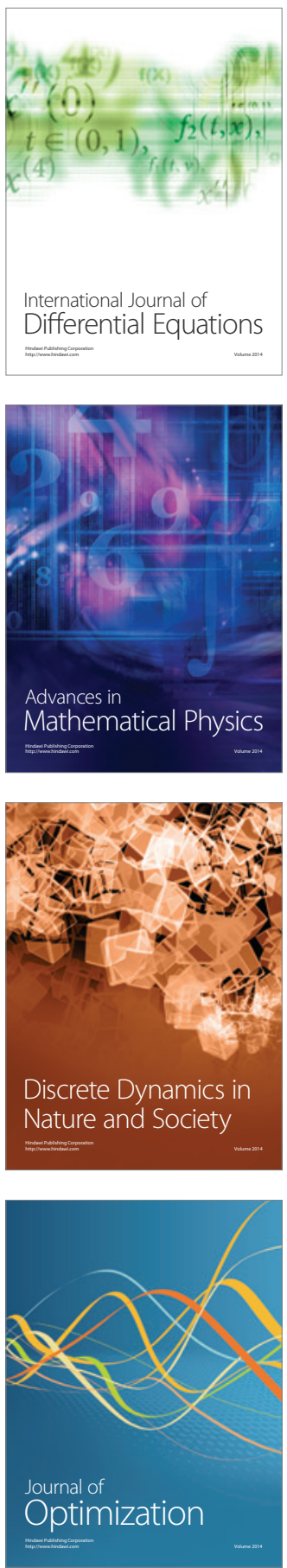